\title{
Externalization of User Model in Mobile Services
}

\author{
http://dx.doi.org/10.3991/ijim.v8i1.3387 \\ Muhammad Asif and John Krogstie \\ NTNU, Trondheim, Norway
}

\begin{abstract}
In most personalized mobile services, the user model remains invisible, and users do not have control over it. Externalization of user models can allow users to get an overview the user model that is used for personalization, and adjust the profile and personalization effects to their needs and preferences. We have evaluated the interactive user model with 42 users, which were exposed to a prototype of interactive user model of personalized news service, for determining whether the proposed externalization, scrutability and privacy privileges were acceptable to the users. The purpose of the study was to find out if it is appropriate to present a user model on the mobile device and to control the sharing of the user model with the service provider. The conclusions show that the users expressed their general approval of the proposed privileges while making useful suggestions regarding improvements to the presentation and interface to the system.
\end{abstract}

Index Terms-Mobile Services, Personalization, Privacy, User Control, User Model.

\section{INTRODUCTION}

The increasing popularity of mobile devices has opened new opportunities for personalized mobile services. There is a variety of personalized mobile services ranging from simple personalized interfaces to more complex contextaware personalized mobile services. User model is a key to personalization which may vary from simple user's demographic data to more detailed information about the user such as context, interests, preferences, expertise, traits etc. User model has become central in a number of classes of systems that are currently of considerable interest: recommender systems, customized documentation, teaching systems, information filtering and other tailored interfaces [1].

In many personalized systems, the user model is considered as purely internal system information, and it is partially or completely hidden from the user [2-4]. Most of the personalized systems are generating user model from implicit feedback from the users' search and browsing history, and explicitly from the classical interfaces that allowed people to express their preferences by browsing along the set of well-defined categories of contents. Users are not only concerned about their private information being used, but also about unintentional flow of information [21]. In UM community, there is a debate on the tradeoff between the user control and the intelligent agents that learn about the user $[16,17]$. A user should be aware of her/his user model used by a system or a service. In addition, user should have control over her/his user model to personalize a mobile service $[15,16]$. Currently, in most personalized systems, the user has no way to discover the details of their user models and the associated personalization [18]. The user model should be visible and accessible to the user so that they may have insight of it.
However, user must be able to view and alter the user model to increase the acceptance of the personalized systems [2].

There is a variety of issues related to the user model such as incorrectness, inconsistency and incompleteness that makes personalized mobile services unacceptable for the users. In addition to this, user has issues of privacy and no control over the user model. We believe that if user models can be externalized on mobile devices, it can alleviate the issues of invisibility and inconsistency. A study [18] has suggested scrutable user models and described it as a basis for understanding and controlling personalization. This approach can be difficult for mobile services due to the inherited constraints of mobile devices. In this study, we wanted to explore if simple user models can be presented on mobile devices and if users are willing to adopt it as a part of mobile service. The proper presentation of a user model on mobile devices can play a key role. The user model should be presented in a simple way so that the user can understand and modify it easily. It can help to make adaptation decisions, to inspect, and modify the values stored in user models. Hiding user models may occlude the system status and hinders control on the adaptation, which might lead to errors, e.g. issuing irrelevant recommendations [2]. However, there is long standing debate about to what extent users should give up control of their interactions [7]. Control on the user model can provide a fine tuning mechanism to obtain a flexible and accurate interest or preferences [8]. Besides this, personalization has also amplified the privacy risks and concerns. Different studies [21-23] have described increasing concerns about privacy in the context of personalization. This problem can be alleviated by giving more control to the users of their user models.

Although there has been research in user modeling, there is a gap between research with the themes of accessibility, ownership, scrutability, and user control; and the practical application of these themes in working prototypes that have been evaluated by end-users [24]. We conducted this study to understand the users' opinion about the externalization of user models along with representation and visualization modalities. The experiment was conducted with a working prototype of an interactive user model of a news service. This is a first study that presented a user model on mobile devices for users to play with and collected users' responses through a post-study assessment questionnaire. We believe that the results of this study will guide the development of future mobile services. Section II describes the motivation and objective along with research questions. Related work about externalization and user modeling are described in section III. Section IV gives an overview of the interactive user model and its elements. The results of the study, discussion and evaluation are described in section V. Section VI concludes the study. 


\section{Motivation AND OBJeCtives}

User has the right to know which information is being shared with service providers. Invisible user models could raise usability issues which can affect the acceptance of the personalized services [16]. The externalization of user models can assist the users to know what information system is utilizing to provide personalization. This can give an opportunity to complete/correct the user models. Moreover, it can facilitate users to have a sense of control over the adaptation of systems by controlling the user model and, the way that the model is interpreted and the way that it used to perform the personalization. It can help people to become more self-aware and avoid selfdeception. Externalization can increase user's understanding of how their user model and feedback contributes to personalization and thereby enhancing their experience of the system. It can also motivate people to share user model data because they feel confident about its meaning and use. Understanding, accepting and trusting a personalized system can improve the user-system interaction [9, 19]. Our research questions in this setting are:

Q1. Do users feel that it is useful to inspect, modify and control their user models?

Q2. Is it appropriate to present a user model in a comprehensive and user friendly way on mobile devices?

The aim is to verify whether the commonly used visual metaphors can present user model by giving a specific user model representation (in this case we are using a user model of personalized mobile news service). We hypothesized that existing visualization modalities or metaphors of mobile services can be used to externalize a user model. This can be verified by providing an interactive user model visualization and see how people feel about it. The goal is to find a way to represent user models to the users and to allow the user to modify her user model on mobile devices. Moreover, the visualization modalities chosen to present the user model can have an impact on the comprehensibility of the user model itself.

\section{EXTERNALIZATION OF USER MODELS AND RELATED WORK}

User model is a key to personalization of mobile services. Currently, the personalization process and user models remain invisible in most of the personalized systems $[2,6]$. Making user models accessible to the users is a key requirement to the acceptance and success of adaptive systems. To ensure acceptance by users, these models need to be scrutable, i.e., users must be able to view and alter them to understand and if necessary correct the assumptions the system makes about the user [2]. Externalization is a first step towards the scrutability of user models. Jameson [10] argued that allowing inspection and parameterization of user models is essential to achieve predictability, transparency, and controllability of an adaptive system. According to Cook and Kay [1], the user needs to be able to understand the provenance of information in her user model, e.g., the user needs to understand why the system believes she is interested in a certain topic. We have not found any research work on externalization of user model related to provision of mobile services so far. Usually, the focus of externalization of user models was on larger systems. In this section, we briefly describe some of the work on externalization of user model and scrutability.
The term scrutability in user modeling signifies that every user's model can be inspected and altered by its owner in order to determine what should be modeled about him/her and how that modeling and following personalization process will be conducted [4]. Introspective views were used to represent user knowledge or interest [2]. According to the authors introspective views can help the user to gain an overview of the entire user model and zoom into a certain part of the model to get a better view on it. Moreover, it can enable the users to filter out unwanted items in order to focus on the relevant ones. A similar study [3] has proposed an approach to control adaptive behavior of the recommender system by allowing users to view and adjust the profile. It also allows users' to see the effects of personalization and modify the interests or preferences accordingly. The um-view interface [1] allows traversing through a user model by expanding the tree of leaves and viewing detailed information about the items in the model. VIUM $[11,12]$ and its successor SIV [12] are capable of visualizing large user models and enable users to get an overview of the whole model, view a subset of related beliefs, filter items by relevance, and obtain detailed information about the displayed items.

Understanding the goal of externalization of user models in mobile services and the means for how to achieve that goal is vital. Here, the goal is to provide reflection and improve the accuracy of the user model. Moreover, the purpose is to provide control to users of their models and improve privacy. SMILI (Open Learner Modeling Framework)[13] has described various issues to consider for externalization of user models. A brief description of the few relevant issues is as following:

- Extent of model accessibility. To what extent the model will be accessible (completely or partially).

- Presentation: How the model will be presented (graphically or textually). In addition, how the model will provide the information (summary, overview, targeted detail or all details).

- Access Initiative: How the model will be accessed. Either the system or user will initiate access.

- Control over accessibility: Who will control (System, User or Others) and how (complete, partial, or none)

- Awareness of effect of model on personalization: How much the user (complete, partial or none) will be aware of the effect of the model on personalization?

- Flexibility of access: How much (complete, partial or none) it is flexible to access the user model.

\section{AN INTERACTIVE USER MODEL}

Externalization of user models provides an opportunity to view and access the user models. Moreover, it can help users to understand the behavior of personalization depending on their models. Therefore, one objective to externalize the user's model is to alleviate the invisibility of user model and personalization process. If the model is simple or easy to understand then the complete access to the user model can improve accuracy and in case of large and complex models, partial access can be more effective[13]. An incorrect user model is a major issue in personalization [18]. We believe that externalization of the user model can enable users to inspect their models and modify if there are any errors. In current systems, users have little or no control of their personal information 
and user models [18]. With the externalization of user models, users can view and manage their own user models, and this can help to give control to the owners of the data.

We believe that by externalizing the user model, the problems of errors in a user model and invisibility can be solved. An interface is an essential part to externalize the user model. Such interfaces are extremely important because users are ignoring or tolerating obvious errors in user models and personalization, as they are obliged to do in more interfaces they use [18]. In addition to viewing the contents of user models, the interface enables users to edit them and to change interest degree. It provides flexible, multidimensional and browse-able user model. The interactive user model has the following capabilities:

\section{A. View}

In this user can view the user model in a variety of ways either in a graphical form or a textual form. This may provide users a chance to reflect on and enhance user knowledge. It can help in raising their awareness of what they do not know. Fig 1 provides an overview of the user model from two perspectives. The first Fig 1(a) gives an overview of the user's interest in particular categories of news. The graphical view gives a summary about the user's news interests which may increase her/his awareness. Moreover, user can view the details of the news interests as well. Fig 1(b) provides an overview of the privacy elements (context and permissions) used by the service. This part describes which context and permissions are utilized by the application. We believe, it can provide a quick overview of privacy elements and can enhance awareness of the privacy.

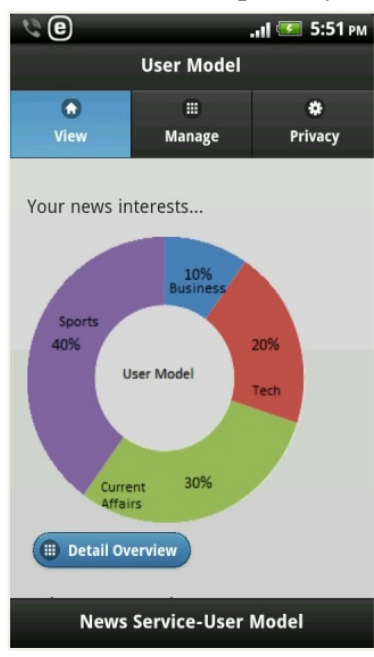

Figure 1. (a) View-Interests

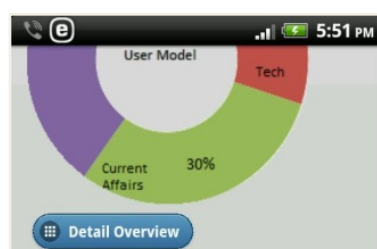

Privacy Overview

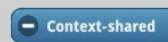

Location, Calendar

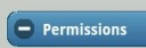

Phone-state, Wifi, Contacts
User Model

(b) View-Privacy

\section{B. Manage}

Control on the user model is becoming the desired feature of externalization. Fig 2 is showing how the user can edit (add, remove and modify) the preferences, interests and other parts of the user model. It provides the user an opportunity to manage their preferences. We wanted to explore whether users will be willing to manage their preferences or interests and how often. Moreover, we wanted to see if it is easy and useful for users to manage preferences explicitly.

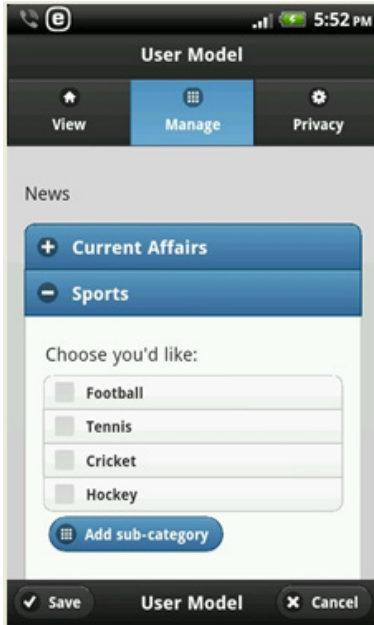

Figure 2. Manage preferences

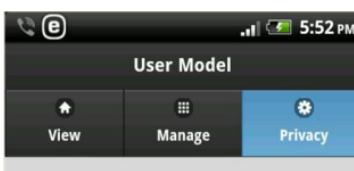

Privacy settings-Context

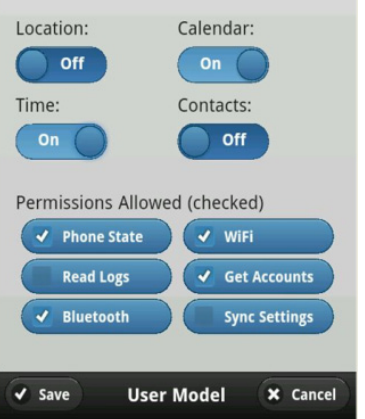

Figure 3. Control Privacy

\section{Privacy}

This can help to manage the privacy of the user model. For example, user can disable his/her location. It can also help to control the personalization by users themselves. Privacy is always a crucial issue in user modeling and privacy components should be included in every UM solution. Users should be given the option to set the status of their information and allowed to decide who can see which part of their user models [4, 14]. Fig 3 is showing two perspectives of privacy context and permissions. The context part is meant to provide an opportunity to the users to control their context. The permission part is representing the permissions used by the service and users have the opportunity to control the permissions used. Here, we wanted to explore how users feel about controlling the privacy.

Through the interactive user model, users can see what they are sharing with the system and how this information is used for personalization. Moreover, users can edit that information and control what they are sharing that can help to understand personalization and control on their privacy. The interactive user model is providing externalization of the user model as well as an opportunity of scrutability of the user model to some extent. It means users can inspect and correct the data that are held about them, and implement privacy policies so that the users can control how their models are accessed and used.

\section{Evaluation AND RESUlts}

To understand the impact and users' perceptions, we have evaluated the approach to externalize the user model of mobile services. In order to do this, we have utilized a working prototype of an interactive user model of a personalized news service and collected 42 responses from a variety of users. Before commencing the test, the participants were given a brief introduction to the prototype of the interactive user model and the services it may provide. A mobile device with the application preinstalled was handed out. The participants were also informed that the data being collected would be part of a research study.

\section{A. Study Design and Procedure}

The study was designed to obtain quantitative feedback regarding the externalization of user model with specific questions mentioned in section II. The experiment consists of three tasks viewing, managing and controlling the 
privacy of the user model of a news service. The participants have to go through each task and provide feedback on the questionnaire provided.

The estimated time of the experiment was $45 \mathrm{mins}$ in which participants have to perform all the three tasks. We handed over three things to the participant at the same time; a mobile device with preinstalled application, a printed user guide and a questionnaire. We have given the flexibility to the participants to fill the questionnaire either after completing each task or after completing all the tasks. All questions in this study are statements to which a user can indicate his/her level of agreement on a fivepoint Likert scale, ranging from -2 (strongly disagree) to +2 (strongly agree); 0 is neutral. The statements in the questionnaire were designed to address the research questions mentioned in the Section II.

\section{B. Participants}

The user study was carried out over a period of three weeks. The study collected 42 users' responses (see Table 1). There were 13 females and 29 males, with $64 \%$ being aged between 25 and 30; and 36\% older. We have seen diverse experience in using mobile services such as $17 \%$ users have less than a year; $45 \%$ users have experience between 2-5 years, and 38\% have more than 5 years' experience in using a different type of mobile services.

TABLE I.

PARTICIPANT DEMOGRAPHICS

\begin{tabular}{|c|c|}
\hline Total & 42 \\
\hline Gender & Female: 13 Male: 29 \\
\hline Age & $25-30$ years: 27 \\
& $>30$ years: 15 \\
\hline Experience in using mobile ser- & $<1$ year: 7 \\
vices & $2-5$ years: 19 \\
& $>5$ years: 16 \\
\hline
\end{tabular}

\section{Results and Discussions}

The whole experiment was divided into three main tasks of viewing, manage and control of user's privacy elements. We have asked 4 questions in each category and received interesting responses as presented below:

\section{Task 1: Viewing the User Model}

We have showed a user' interests of personalized news services to the users on a mobile device and asked if it was easy to understand/view it on a mobile device. We got $29 \%$ neutral responses on this question; and $50 \%$ users were agree that user model is easy to view and understandable on the mobile device, and $21 \%$ users strongly agreed that it is easy to view the model, and it is understandable. We did not get any disagreement on this. The second question was if it was easy to keep track of preferences/interests. We got $64 \%$ agreement on this and $36 \%$ of the users strongly agreed. The third question was asked specifically on if it was easy to keep track of privacy elements, and we got $67 \%$ agreement and 33\% strong agreement on this which shows that it was not difficult for users to keep track of privacy elements when needed. The last question was about if it was useful to know the permissions used by the service. We got mixed responses here: $12 \%$ users did not know show agreement or disagreement on this while $62 \%$ marked agree and $26 \%$ marked strongly agree that it was not difficult to view permissions used by the service. The results on this part is summarized in Fig. 4
Task 1: Viewing the User Model

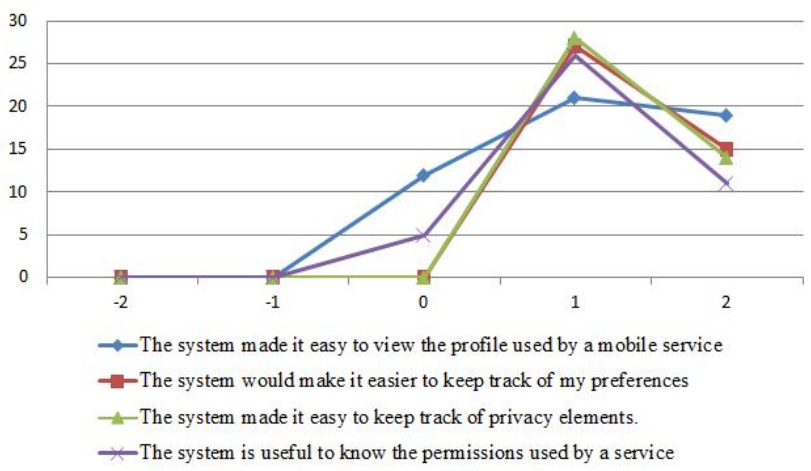

Figure 4. Viewing the User Model

\section{Task 2: Managing the User Model}

In task 2, users were asked four questions related to edit or modifying their users' models which includes interests or preferences of news service. We asked the users if it is easy to modify or correct the values in the user model. We got diverse response, $21 \%$ users neither agreed nor disagreed, 43\% marked agree, and 29\% marked strongly agree. However, we have seen that $9 \%$ were disagreeing with the easiness of modifying the interests. The second question was about managing the overall profile, and $31 \%$ gave neutral response. $41 \%$ agreed, and $28 \%$ strongly agreed on that it was easy to manage the profile on the mobile device. The next question was about the interface if it was intuitive and clear to understand. The agreed response was $41 \%$, and $22 \%$ were strongly agreed. We got $37 \%$ neutral response about the intuitiveness of the user interface to manage the profile. The last question in this category was about if the system was useful to control the preferences shared with the service. We got 38\% agreed and $39 \%$ strongly agreed response on controlling the preferences. However, $23 \%$ gave neutral response on this. From the results, it is revealed that interface for managing the profile required improvements to make it understandable and easy to use. The results on this part is summarized in Fig. 5

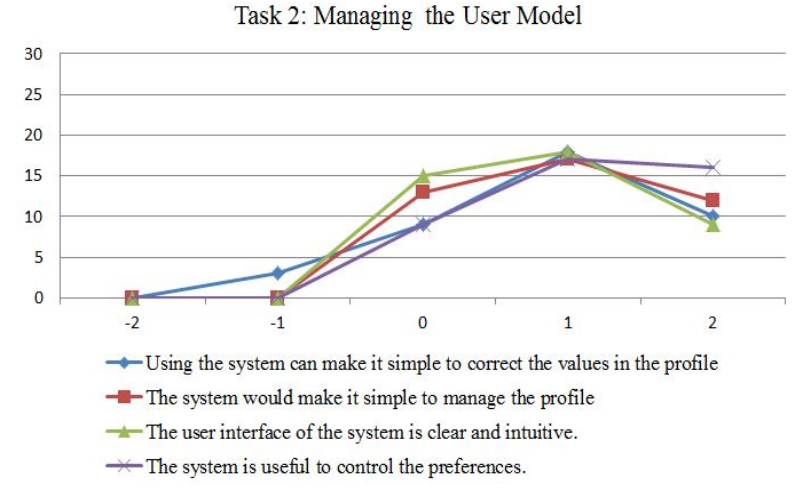

Figure 5. Managing the User Model

\section{Task 3: Privacy of the User Model}

The last task was about the viewing and managing the privacy elements of the user model. We asked four questions related to control the privacy of the context and permissions elements used by the service. The first question was to ask if users are willing to control the privacy elements on the mobile device. We have seen that $22 \%$ 
agreed, and $88 \%$ strongly agreed to take control of privacy elements shared with the service. The second question was if the system was useful to manage the context elements (location, time, etc.) shared with the service. $29 \%$ of the users agreed, and $71 \%$ strongly agreed that it was easy to manage and control the context elements. The next question was specifically about if it was easy to manage the permissions and 33\% agreed and 67\% strongly agreed on this. The last question was about the overall usefulness of the system to control the privacy elements (context and permissions). Results showed that $36 \%$ users agreed, and $64 \%$ strongly agreed on the usefulness of the system to control and manage the privacy elements. The results from this part are summarized in Fig. 6.

Task 3: Privacy of the User Model

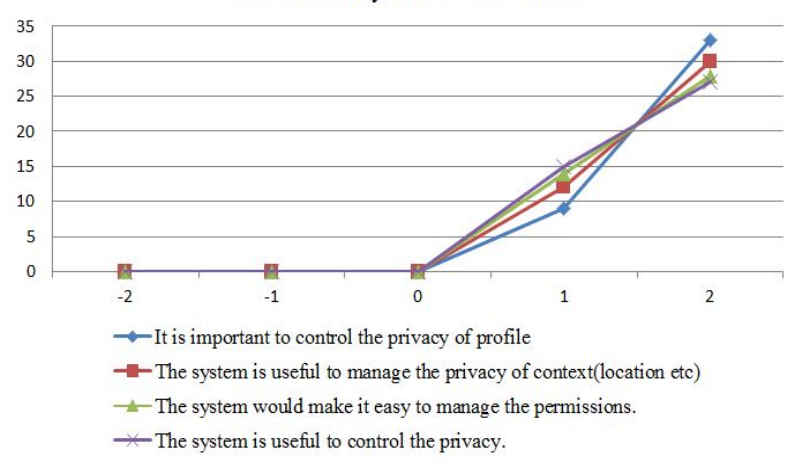

Figure 6. Privacy of the User Model

The first research question was to know if users really feel that it is useful to inspect, modify and control their user models. If we look at the results of task 1(see Figure 4 ), it shows that users are willing to inspect their user models. Users showed great interest in inspecting the preferences and privacy elements shared with a mobile service. It can also be observed that users were at ease to use the system. Similarly, the second task was if users really feel comfortable to modify their interests or preferences. We received a considerable positive response on that users are willing to manage or correct their preferences. Only 9\% disagreed on this (see Figure 5). The third task was to know if users are interested in controlling privacy elements (context and permissions). If we look at the results (Figure 6), it can be observed that users strongly agreed to take the control of the privacy elements they shared. The second research question was to know if it is appropriate to present a user model in a comprehensive and user friendly way on mobile devices. We have presented a user model with very few elements and used common GUI elements to present it. The results showed that it was not difficult for users even with limited experience in the use of mobile services to handle such kind of user model on the mobile device (see Section V). Although, the presentation of the user model was not intuitive, users liked the idea to have user model under their control.

The main focus of the study was to find the subjects opinion about the possibility to inspect, modify and taking control of their user model. From the results, it is revealed that users agreed to view, manage and control the privacy of their user model. Moreover, it is evident that users strongly agreed on taking control of their privacy elements and willing to manage their user models. The second objective was to find out if it is appropriate to present a user model on a mobile device. Although, we used common GUI elements to present the user model but still there were responses on the difficulty of using it or subjects did not give any response. It is revealed that the interface requires more improvements to make it more understandable, intuitive and easy to use. On the other hand, it is critical to know which elements of the user model should be externalized and what should be the main objective of the externalization.

\section{CONCLUSIONS}

Most approaches to scrutable user modeling have primarily focused on allowing users to view information stored in the model. Little research has been conducted to know the perception of users to provide control over the user model of mobile services. The ownership, accessibility, scrutability and user control are the important concerns of user modeling. We have conducted this study to learn how users respond to our visual displays of a user model on a mobile device. We describe an experimental evaluation that seeks to answer if it is appropriate to present user model of a service on a mobile device in a controlled lab situation using a running prototype of an interactive user model. It was found that users are willing to control a user model of a mobile service, particularly the privacy elements. It was also found that externalization of the user model can help the users to increase their awareness and control of their user model. However, this study has a limitation that the user model presented contains few elements. There is a need to do another study to verify the idea with large user model of a mobile service. This is an ongoing work in connection to mobile application and services in the Wireless Trondheim Living Lab [25].

\section{REFERENCES}

[1] Cook, R. and J. Kay, The justified user model: a viewable, explained user model. 1994: Basser Department of Computer Science, University of Sydney.

[2] Bakalov, F., et al., Introspectiveviews: An interface for scrutinizing semantic user models, in User Modeling, Adaptation, and Personalization. 2010, Springer. p. 219-230. http://dx.doi.org/10.1007/978-3-642-13470-8 21

[3] Bakalov, F., et al., An Approach to Controlling User Models and Personalization Effects in Recommender Systems. 2013.

[4] Kyriacou, D., H.C. Davis, and T. Tiropanis, Evaluating Three Scrutability and Three Privacy User Privileges for a Scrutable User Modelling Infrastructure, in User Modeling, Adaptation, and Personalization. 2009, Springer. p. 428-434. http://dx.doi.org/10.1007/978-3-642-02247-0_48

[5] Asif, M. and J. Krogstie. Taxonomy of Personalization in Mobile Services. Proceedings of the 10th IADIS International Conference e-Society, 2012.

[6] Asif, M. and J. Krogstie. Mobile Client-side Personalization. International Conference on Privacy and Security in Mobile Systems. PRISMS 2013, Global Wireless Summit, IEEE, NJ, USA.

[7] Shneiderman, B. and P. Maes, Direct manipulation vs. interface agents. interactions, $1997 . \quad 4(6)$ : p. 42-61. http://dx.doi.org/10.1145/267505.267514

[8] Esteban, A.D., P.G. Gómez-Navarro, and A.G. Jiménez, Evaluating a user-model based personalisation architecture for digital news services, in Research and Advanced Technology for Digital Libraries. 2000, Springer. p. 259-268. http://dx.doi.org/10.1007/3-540-45268-0 24

[9] Vernero, F., et al., An Experiment to Evaluate how to Better Present User Models to the Users. UMAP 2009, 2009: p. 21. 
[10] Jameson, A., Adaptive interfaces and agents. Human-Computer Interaction: Design Issues, Solutions, and Applications, 2009. 105.

[11] Uther, J. and J. Kay, VIUM, a web-based visualisation of large user models, in User Modeling 2003. 2003, Springer. p. 198-202. http://dx.doi.org/10.1007/3-540-44963-9_26

[12] Kay, J. and A. Lum. Building user models from observations of users accessing multimedia learning objects, in Adaptive multimedia retrieval. 2004, Springer. p. 36-57. http://dx.doi.org/10.1007/978-3-540-25981-7 3

[13] Bull, S. and J. Kay. Student Models that Invite the Learner In: The SMILI:Open Learner Modelling Framework. International Journal of Artificial Intelligence in Education, 2007. 17(2): p. 89-120.

[14] Kyriacou, D. and H.C. Davis. Moving towards life-long User Modeling. in Advanced Learning Technologies, 2008. ICALT'08. Eighth IEEE International Conference on. 2008. IEEE.

[15] Asif, M. and J. Krogstie. "Mobile Services Personalization Evaluation Model." International Journal of u- and e- Service, Science and Technology 6(2): 1-12.

[16] Asif, M. and J. Krogstie.Research Issues in Personalization of Mobile Services. International Journal of Information Engineering and Electronic Business Vol 4. Issue 4.

[17] Jones, N., P. Pu, and L. Chen, How users perceive and appraise personalized recommendations, in User Modeling, Adaptation, and Personalization. 2009, Springer. p. 461-466. http://dx.doi.org/10.1007/978-3-642-02247-0 53

[18] Kay, J. and B. Kummerfeld. Creating personalized systems that people can scrutinize and control: Drivers, principles and experience. ACM Transactions on Interactive Intelligent Systems (TiiS), 2012. 2(4): p. 24. http://dx.doi.org/10.1145/239512 $\underline{3.2395129}$

[19] Asif, M. and J. Krogstie. Role of Personalization in Mobile Services Adoption. Proceedings of the International Conference on Multimedia and Human Computer Interaction. International ASET, 2013.

[20] Asif, M. and J. Krogstie. Mobile student information system, Campus-Wide Information Systems 28(1): 5-15, 2011.

[21] Toch, E., Y. Wang, et al. (2012). Personalization and privacy: a survey of privacy risks and remedies in personalization-based systems. User Modeling and User-Adapted Interaction 22(1-2): 203-220. http://dx.doi.org/10.1007/s11257-011-9110-z
[22] Anton, A.I., Earp, J.B., Young, J.D.: How internet users' privacy concerns have evolved since 2002. IEEE. Secur. Priv. 8(1), 21-27 2010. http://dx.doi.org/10.1109/MSP.2010.38

[23] Turow, J., King, J., Hoofnagle, C.J., Bleakley, A., Hennessy, M.: Americans reject tailored advertising and three activities that enable it. 2009.

[24] Wasinger, R., J. Wallbank, et al. (2013). Scrutable User Models and Personalised Item Recommendation in Mobile Lifestyle Applications. User Modeling, Adaptation, and Personalization, Springer: $77-88$

[25] is S. Andresen, J. Krogstie, and T. Jelle. Lab and research activities at wireless trondheim. In Proc. of the 4th IEEE International Symposium on Wireless Communication Systems (ISWCS'07), 2007

\section{AUTHORS}

Muhammad Asif is a $\mathrm{PhD}$ student in the Department of Computer and Information Science (IDI), Norwegian University of Science and Technology (NTNU), Norway. He completed his master degrees in Information System Engineering in 2010 at Norwegian University of Science and Technology. His research interests include mobile information systems, mobile computing, user modeling, and model driven information systems engineering. (email: muhammad.asif@idi.ntnu.no).

John Krogstie holds a PhD (1995) and an MSc (1991) in information systems from the Norwegian University of Science and Technology (NTNU), where he is currently a full professor in Information Systems. John Krogstie is the Norwegian representative for IFIP TC8 and Chair of IFIP WG 8.1 on information system design and evaluations. He has published around 200 refereed papers in journals, books and archival proceedings since 1991. (e-mail: krogsite@idi.ntnu.no).

Submitted 21 November 2013. Published as re-submitted by the authors 05 January 2014. 